\title{
MicroRNA-15a-5p serves as a potential biomarker and regulates the viability and apoptosis of hippocampus neuron in children with temporal lobe epilepsy
}

\author{
$\mathrm{Na} \mathrm{Li}{ }^{1 \dagger}$, Jingmei Pan ${ }^{1+}$, Wei Liu', Yuanyuan $\mathrm{Li}^{2}$, Feng $\mathrm{Li}^{3^{*}}$ and Min Liu${ }^{4^{*}}$
}

\begin{abstract}
Background: Temporal lobe epilepsy (TLE) is the most common type of epilepsy, usually starting in childhood. The dysregulation of microRNAs (miRNAs) has been identified in neurological disorders. The current study investigated the expression level and clinical significance of miR-15a-5p in TLE children, and explored its function in regulating cell behaviors of hippocampal neurons.

Methods: The expression level of miR-15a-5p was examined in the serum of 63 TLE children. Primary hippocampal cells were cultured in magnesium-free medium to mimic TLE condition in children. The effect of miR-15a-5p on the viability and apoptosis of hippocampus neuron was assessed using MTT or flow cytometric apoptosis assay.

Results: TLE children had significantly low expression of miR-15a-5p. MiR-15a-5p was of great value for the diagnosis of TLE in children, with high specificity and sensitivity. The expression level of miR-15a-5p was decreased significantly in hippocampal cells treated in the magnesium-free medium. Overexpression of miR-15a-5p attenuated TLE-induced reduction for cell viability, and reversed the cell apoptosis induced by TLE.

Conclusions: MiR-15a-5p is downregulated in children with TLE, and overexpression of miR-15a-5p promoted the viability and inhibited the apoptosis of hippocampal neuron. MiR-15a-5p may be a promising biomarker for the diagnosis of children TLE.
\end{abstract}

Keywords: miR-15a-5p, Temporal lobe epilepsy, Hippocampus neuron, Viability, Apoptosis

\section{Introduction}

Epilepsy is a common neurological disorder, and is defined as seizures that occur as a result of neuronal discharge in a certain region of the brain [1]. According to statistic, the incidence of epilepsy in children ranges from 33 to 82 per 100,000 children per years [2]. Temporal lobe epilepsy (TLE) is the most common type of

\footnotetext{
*Correspondence: lifeng12_12@163.com; minliuc5236@163.com

${ }^{+} \mathrm{Na} \mathrm{Li}$ and Jingmei Pan contributed equally to this work.

${ }^{3}$ Department of Emergency Surgery, Weifang People's Hospital, No. 151, Guangwen Street, Weifang, Shandong 261000, P.R. China

${ }^{4}$ Department of Medical Insurance, Weifang Maternal and Child Health Care Hospital, Weifang, Shandong 261021, P.R. China

Full list of author information is available at the end of the article
}

focal epilepsy with seizure onset from the temporal lobe structure [3]. Unfortunately, children with TLE are often difficult to control with one medication, and two or more appropriate medications are always demanded [4]. The goal of treatment for epilepsy is to reduce the duration of seizures, which is related to neuroprotection. Neuroprotection is considered to be an important aspect of preventing the development of epilepsy.

MicroRNAs (miRNAs) are small noncoding RNAs, which function to regulate multiple developmental processes at the posttranscriptional level. A series of reports have proved the importance of miRNAs dysregulation in

(c) The Author(s). 2020 Open Access This article is licensed under a Creative Commons Attribution 4.0 International License, which permits use, sharing, adaptation, distribution and reproduction in any medium or format, as long as you give appropriate credit to the original author(s) and the source, provide a link to the Creative Commons licence, and indicate if changes were made. The images or other third party material in this article are included in the article's Creative Commons licence, unless indicated otherwise in a credit line to the material. If material is not included in the article's Creative Commons licence and your intended use is not permitted by statutory regulation or exceeds the permitted use, you will need to obtain permission directly from the copyright holder. To view a copy of this licence, visit http://creativecommons.org/licenses/by/4.0/ The Creative Commons Public Domain Dedication waiver (http://creativecommons.org/publicdomain/zero/1.0/) applies to the data made available in this article, unless otherwise stated in a credit line to the data. 
the nervous system, and have brought growing body of evidence on miRNA dysregulation in neurological disorders, such as Parkinson's disease and Alzheimer's disease $[5,6]$. A number of recently published studies have now profiled miRNA expression in experimental models of status epilepticus, and multiple abnormally expressed miRNAs have been identified [7-9]. A major study has determined that circulating miR-15a-5p is aberrantly expressed in adult epilepsy patients [10]. Additionally, miR-15a-5p is also suggested to be at low expression in patients with Alzheimer's disease, which is closely related to the development of epilepsy $[11,12]$. These evidence demonstrate the crucial role of miR-15a-5p in the pathogenesis of neurological disorders. However, the expression of miR-15a-5p in children with TLE is still unclear, because TLE in children is different from the syndrome observed in adults [4].

Therefore, in the presents study, we investigated the expression and clinical value of miR-15a-5p in children with TLE, and further explored its effect on hippocampal neuron viability and apoptosis in a TLE cell model.

\section{Materials and methods}

\section{Study population and sample collection}

Sixty-three children with TLE were collected with the mean age of $9.81 \pm 2.79$ years old, who were diagnosed according to their clinical seizure semiology, videoelectroencephalography (EEG) monitoring, and highresolution magnetic resonance imaging (MRI) results. According to EEG results, the patients manifested repetitive spikes and spike-and-wave discharges in the left and/or right temporal lobes. According to the MRI results, the abnormal MRI imaging includes tumors, disorders of neuronal migration and cortical organization, vascular malformations, mesial temporal sclerosis, and neocortical sclerosis attributable to brain injury. Patients who had non-epileptic disorders were excluded from the study, including electrolyte disturbances, metabolic disorders, acute brain disease or trauma, and non-epileptic paroxysmal events mimicking epilepsy. Sixty-seven healthy individuals were recruited as the control group, with the mean age of $10.13 \pm 2.46$ years old. $5 \mathrm{ml}$ peripheral blood samples were collected from each participant and centrifuged immediately, then the serum samples were stored at $-80^{\circ} \mathrm{C}$ for further analysis.

The protocol of this study was approved by the Ethics Committee of Yidu Central Hospital of Weifang, and written informed consent was collected from each participant.

\section{Primary culture of hippocampal neurons from newborn rats}

Wistar rats were purchased from the Shanghai Animal Laboratory Center, and were housed in cages with a constant indoor temperature of $25^{\circ} \mathrm{C}$ and had free access to food and water. All animals were treated according to the Guide for the Care and Use of Laboratory Animals of the Institute for Laboratory Animal Research. One male rat was housed with a female rat for the generation of newborn rats. As described in previous research, primary hippocampal cells were collected from P0 newborn rats and cultured [13]. Briefly, three slices of hippocampus were collected from the brain and then were digested with $0.5 \%$ Trypsin at $37^{\circ} \mathrm{C}$ for $15 \mathrm{~min}$. Then the dissociated single cells were washed twice with Hank's buffered saline solution. After treatment with DNase I, neurons were placed on glass coverslips with plating medium and cultured in a humidified incubator with $5 \% \mathrm{CO}_{2}$ at $37^{\circ} \mathrm{C}$. After confirming that the cells are viable and have attached, cells were cultured in Neurobasal medium (Invitrogen; Thermo Fisher Scientific, Inc., Waltham, MA, USA). Once a week, replace onethird of the medium with fresh Neurobasal medium. After incubation for 8 days, primary hippocampal neurons were used for subsequent experiment.

\section{Establishment of epileptiform discharge model in rat hippocampal neurons}

To mimic TLE condition in children, hippocampal neurons from newborn rats were cultured in magnesiumfree medium (145 g NaCl, $2.5 \mathrm{~g} \mathrm{KCl}, 10 \mathrm{~g}$ HEPES, $2 \mathrm{~g}$ $\mathrm{CaCl} 2,10 \mathrm{~g}$ glucose, $0.002 \mathrm{~g}$ glycine dissolved in 1000 $\mathrm{mL}$ distilled water, $\mathrm{pH}$ 7.2) at $37^{\circ} \mathrm{C}$ for $3 \mathrm{~h}$, and the hippocampal epileptiform activity was induced $[14,15]$.

\section{Cell transfection}

MiR-15a-5p mimic and its negative control (mimic NC) were provided by Gene-Pharma (Shanghai, China). Rat primary hippocampal neurons were plated on a 96 well plate, and cell transfection was performed at a confluency of $85-90 \%$. According to the transfection, cells were assigned into four groups: control group (untreated cells), No $\mathrm{Mg}^{2+}$ (cells were cultured in a magnesium-free medium), No $\mathrm{Mg}^{2+}+$ mimic NC (cells were transfected with miR-15a-5p mimic NC, $24 \mathrm{~h}$ post-transfection cells were cultured in a magnesium-free medium at $37^{\circ} \mathrm{C}$ for $3 \mathrm{~h}$ ), No $\mathrm{Mg}^{2+}+$ miR-15a-5p mimic (cells were transfected with miR-15a-5p mimic, $24 \mathrm{~h}$ post-transfection cells were cultured in a magnesium-free medium at $37^{\circ} \mathrm{C}$ for $3 \mathrm{~h}$ ). Lipofectamine 2000 (Invitrogen, Carlsbad, CA, USA) was used for the transfection according to the manufacturer's protocols. $24 \mathrm{~h}$ post-transfection, cells were cultured in a magnesium-free medium at $37^{\circ} \mathrm{C}$ for $3 \mathrm{~h}$ to establish TLE model. The control group was treated with nonmagnesium-free medium, which is magnesium-free medium with $1 \mathrm{mM} \mathrm{MgCl}$. 
RNA extraction and quantitative real-time polymerase chain reaction ( $q R T-P C R$ )

Total RNA was isolated from both human serum and cultured rat hippocampal neurons by using Trizol reagent (Invitrogen, Carlsbad, CA, USA) based on the manufacturer's protocol. Reverse transcription was performed using TaqMan miRNA reverse transcription kit (Applied Biosystems, Foster City, CA, USA). qRT-PCR assay was performed to determine the gene expression using a SYBR Premix Ex Taq ${ }^{\mathrm{Tm}}$ II commercial kit (Takara, Dalian, China) and the Applied Biosystems 7900 RealTime PCR System (Applied Biosystems, Foster City, $\mathrm{CA})$. The following thermocycling conditions were used for the PCR: initial denaturation at $95^{\circ} \mathrm{C}$ for $5 \mathrm{~min} ; 30$ cycles of $95^{\circ} \mathrm{C}$ for $30 \mathrm{~s}, 60^{\circ} \mathrm{C}$ for $30 \mathrm{~s}$ and $72^{\circ} \mathrm{C}$ for $20 \mathrm{~s}$; and a final extension at $72^{\circ} \mathrm{C}$ for $10 \mathrm{~min}$. The relative gene expression was normalized to that of the internal control U6 according to the comparative delta CT $\left(2^{-\Delta \Delta \mathrm{Ct}}\right)$ method. The primers used were as follows: miR-15a-5p forward, 5'-GGGUAGCAGCACAUAAU GG-3' and reverse, 5' -CTCAACTGGTGTCGTGGA-3'; and U6 forward, 5' -CTCGCTTCGGCAGCACA-3' and reverse, 5' -AACGCTTCACGAATTTGCGT-3'.

\section{MTT assay}

The cell viability was assessed using MTT assay according to the previous research [16]. $50 \mu \mathrm{l}$ of MTT (SigmaAldrich; Merck, Darmstadt, Germany) was added into each well and incubated for further $3 \mathrm{~h}$, followed by addition of $150 \mu \mathrm{l}$ of dimethyl sulfoxide (DMSO) (Sigma-Aldrich; Merck). The cell viability was determined by measuring the absorbance at $490 \mathrm{~nm}$ using a microplate reader.

\section{Flow cytometric apoptosis assay}

An Annexin V-FITC Apoptosis Detection kit (Keygen Biotechnology) was used to detect cell apoptosis. Cells of each group were harvest, and washed with incubation buffer $(10 \mathrm{mmol} / \mathrm{L} \quad \mathrm{HEPES} / \mathrm{NaOH}, \mathrm{pH} 7.4,140$ $\mathrm{mmol} / \mathrm{L} \mathrm{NaCl}, 5 \mathrm{mmol} / \mathrm{L} \mathrm{CaCl}_{2}$ ). Subsequently, the cells were re-suspended with $\mathrm{PBS}$ and mixed with $5 \mu \mathrm{l}$ Annexin V-FITC and PI staining solution. After incubation for 10-15 min in the dark, SA-FLOUS was added and incubated in the dark for another $20 \mathrm{~min}$. The apoptotic rates were measured using a FACSCalibur flow cytometer (BD Biosciences).

\section{Statistical analysis}

All the data analyses were performed using SPSS version 18.0 software (SPSS Inc., Chicago, IL) and GraphPad Prism 5.0 software (GraphPad Software, Inc., USA). The data were expressed as mean \pm standard deviation (SD). Demographic data were analyzed using an independentsamples t-test or a chi-squared test. Differences between two groups were compared using student's $t$-test or oneway ANOVA analysis. Receiver operating characteristic (ROC) curve analysis was applied to determine the specificity and sensitivity of miR-15a-5p levels regarding the diagnosis of TLE. Each experiment had at least three repetitions. $P<0.05$ was considered to be statistically significant.

\section{Results}

The demographic and clinical characteristics of the study population

The demographic and clinical characteristics of both case and control groups were summarized in Table 1. The age of the individuals ranged from 6 to 14 years old in the healthy controls group, and 5-15 years in the TLE patients group. There was no significant difference for the age and gender distribution between the control and TLE patients group $(P>0.05$, Table 1$)$.

\section{MiR-15a-5p expression level in the serum of children with TLE}

The expression level of miR-15a-5p in the serum of children with TLE was estimated using qRT-PCR. It was noted that compared with the control group, the TLE children had significantly low expression of miR-15a-5p $(P<0.001$, Fig. 1$)$, suggesting the potential role of miR$15 a-5 p$ in the pathogenesis of TLE. Furthermore, the expression level of miR-15a-5p was compared in the clinical groups of TLE patients according to the results of EEG and MRI. As shown in Fig. 1b, there was no significance for miR-15a-5p expression in bilateral temporal patients compared with unilateral temporal patients $(P>0.05)$.

Table 1 Clinical characteristics of the patients with TLE and the healthy controls

\begin{tabular}{|c|c|c|c|}
\hline Parameters & $\begin{array}{l}\text { Healthy controls } \\
(n=67)\end{array}$ & $\begin{array}{l}\text { TLE patients } \\
(n=63)\end{array}$ & $P$-value \\
\hline Age (years) & $10.13 \pm 2.46$ & $9.81 \pm 2.79$ & 0.482 \\
\hline \multicolumn{4}{|l|}{ Gender } \\
\hline Male & 35 & 33 & 0.987 \\
\hline Female & 32 & 30 & \\
\hline \multicolumn{4}{|l|}{ Interictal EEG } \\
\hline Unilateral temporal & - & 47 & \\
\hline Bilateral temporal & - & 16 & \\
\hline \multicolumn{4}{|l|}{ Epilepsy type } \\
\hline Simple partial & - & - & \\
\hline Complex partial & - & 63 & \\
\hline \multicolumn{4}{|l|}{ Brain MRI finding } \\
\hline No abnormality & - & 52 & \\
\hline Mesial sclerosis & - & 11 & \\
\hline
\end{tabular}

$T L E$ temporal lobe epilepsy, EEG electroencephalography, MRI magnetic resonance imaging 

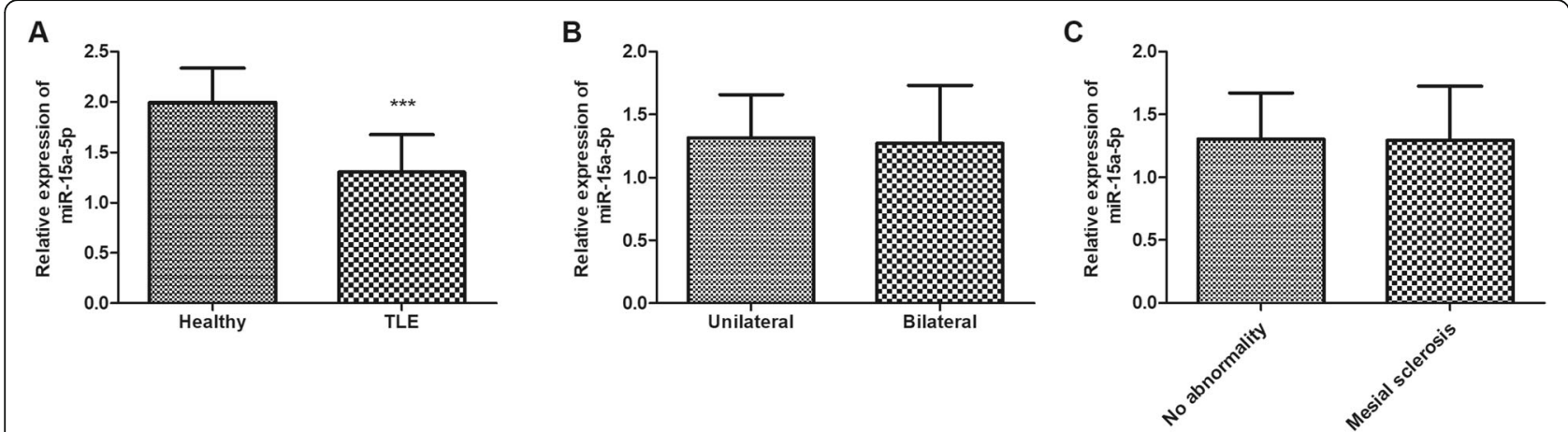

Fig. 1 The expression level of miR-15a-5p in the serum of children with temporal lobe epilepsy (TLE) and healthy controls. a. The TLE children had significantly low expression of miR-15a-5p compared with healthy group. $\mathbf{b}$. There was no significance for miR-15a-5p expression in bilateral temporal patients compared with unilateral temporal patients. $\mathbf{c}$. There was no significance for miR-15a-5p expression in mesial sclerosis patients compared with no abnormality patients. ${ }^{* *} P<0.001$

Besides, similar result was also observed in no abnormality and mesial sclerosis groups $(P>0.05$, Fig. 1c). These data indicated that miR-15a-5p expression did not vary from different subgroups of TLE patients.

\section{The diagnostic value analysis of serum miR-15a-5p for}

TLE

A ROC curve was conducted to calculate the diagnostic value of serum miR-15a-5p for TLE. As shown in Fig. 2, the area under the curve (AUC) for miR-15a-5p was 0.908 , with a sensitivity of $82.5 \%$ and specificity of $88.1 \%$ at the cutoff value of 1.650 . These data revealed that miR-15a-5p might be a sensitive biomarker to distinguish TLE patients from healthy individuals.

\section{Expression level of miR-15a-5p in TLE cell model}

The TLE cell model was established to explore the expression level of miR-15a-5p in vitro. To mimic TLE

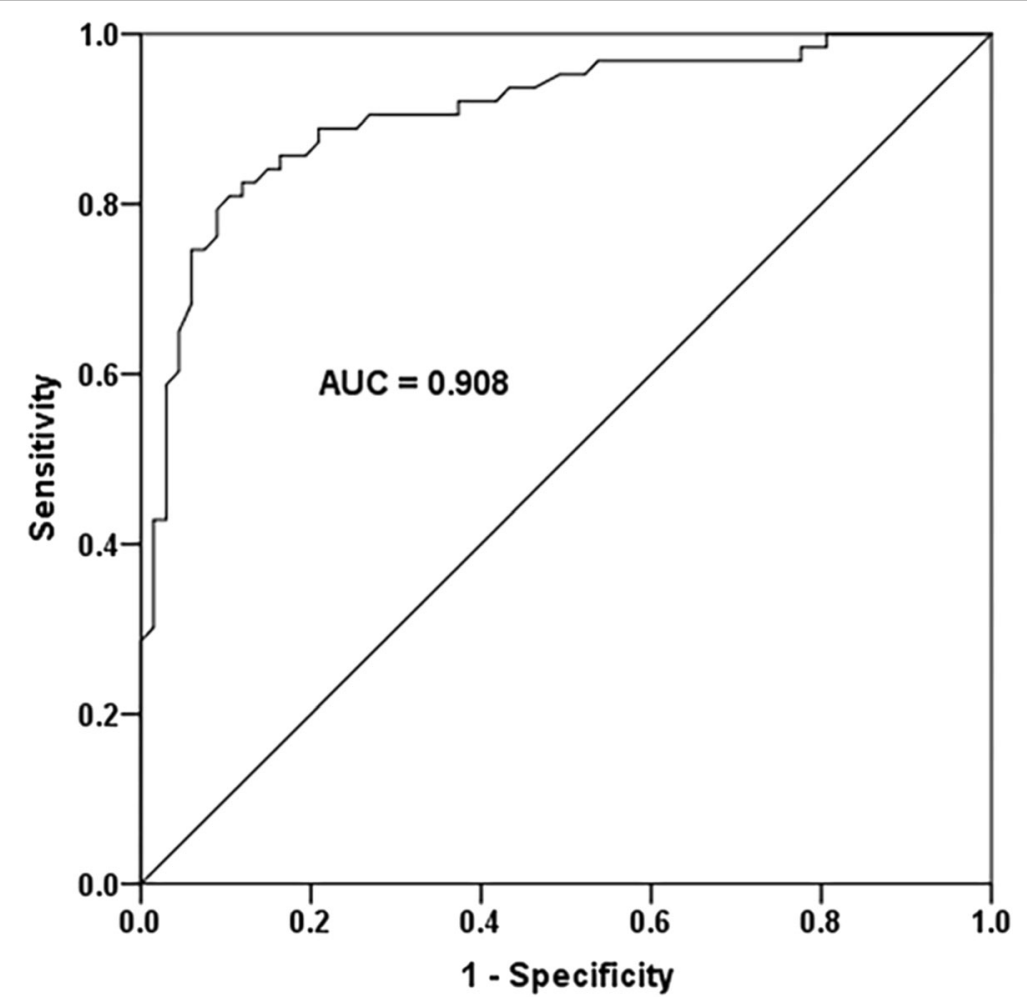

Fig. 2 A receiver operating characteristic ROC curve was conducted to calculate the diagnostic value of serum miR-15a-5p for TLE. The area under the curve (AUC) for miR-15a-5p was 0.908 , with a sensitivity of $82.5 \%$ and specificity of $88.1 \%$ at the cutoff value of 1.650 


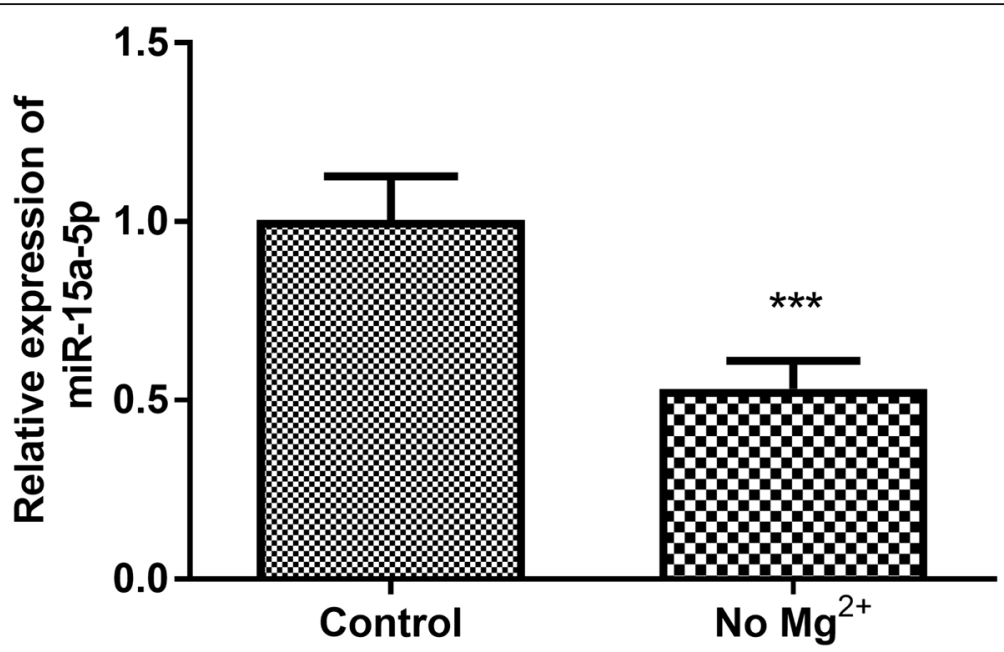

Fig. 3 To mimic TLE condition in children, primary hippocampal cells were collected from newborn rats, and cultured in the magnesium-free medium for $3 \mathrm{~h}$ to establish TLE cell model. The expression level of miR-15a-5p was decreased significantly in hippocampal cells treated in magnesium-free medium. ${ }^{* *} P<0.001$

condition in children, primary hippocampal cells were collected from newborn rats, and cultured in the magnesium-free medium for $3 \mathrm{~h}$. As shown in Fig. 3, compared with the control group, the expression level of miR-15a-5p was decreased significantly in hippocampal cells treated in magnesium-free medium $(P<0.001)$.

\section{Effects of miR-15a-5p on cell viability and apoptosis}

To explore the role of miR-15a-5p in children with TLE, cell transfection was performed to regulate the expression level of miR-15a-5p in hippocampal cells. The overexpression efficiency was examined by using qRT-PCR, and the results suggested that transfection of miR-15a$5 \mathrm{p}$ mimic lead to a remarkable increase in the expression level of miR-15a-5p (Fig. 4a, $P<0.001$ ). It was found that compared with the control group, the viability of hippocampal cells treated in the magnesium-free medium was significantly reduced, while the cell apoptosis was remarkably increased $(P<0.001)$. As shown in Fig. 4 b, transfection with miR-15a-5p mimic significantly attenuated TLE-induced reduction for cell viability $(P<0.001)$. Additionally, overexpression of miR-15a-5p also attenuated TLE-induced cell apoptosis (Fig. 4b, $P<0.001$ ).

\section{Discussion}

TLE is one of the most common and intractable forms of epilepsy, which is characterized by recurring seizures due to abnormal neuronal excitability, usually starting in childhood. It has been proved that brain may be at greater risk for epilepsy attack early in life, and the mechanism for the
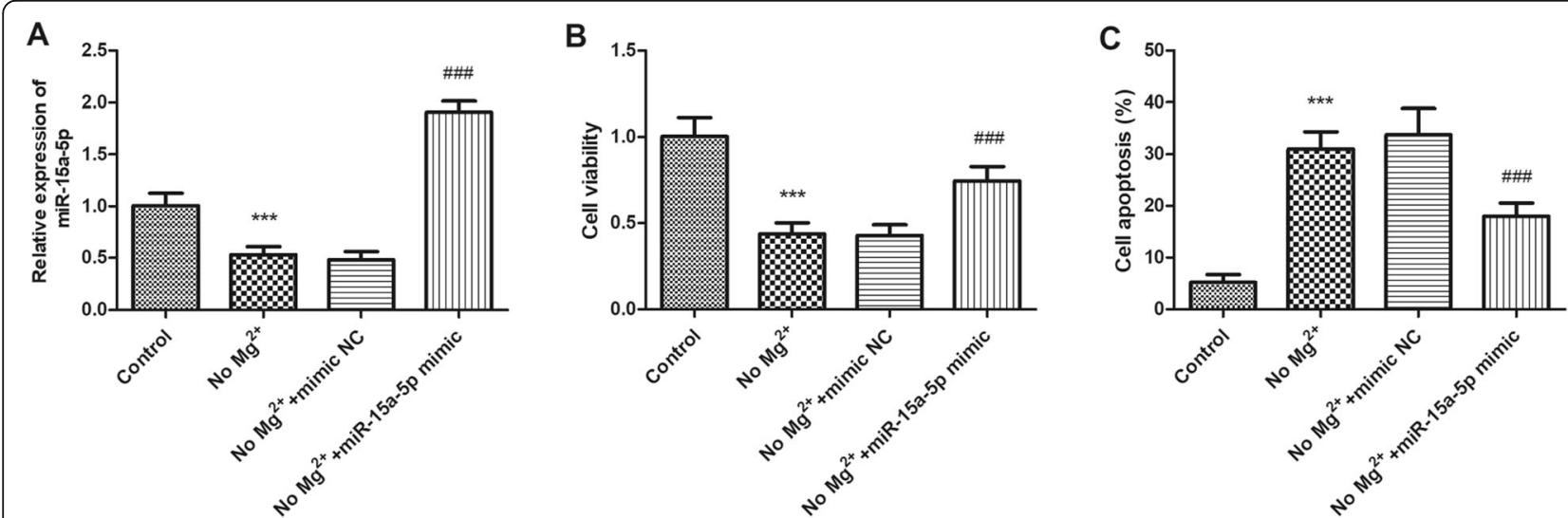

Fig. 4 The effect of miR-15a-5p on the viability and apoptosis of hippocampal neuron. $\mathbf{a}$. Transfection of miR-15a-5p mimic lead to a remarkable increase in the expression level of miR-15a-5p. b. The viability of hippocampal cells treated in the magnesium-free medium was significantly reduced which was reversed by miR-15a-5p mimic transfection. $\mathbf{c}$. The cell apoptosis of hippocampal cells treated in the magnesium-free medium was significantly increased, overexpression of miR-15a-5p attenuated TLE-induced cell apoptosis. ${ }^{* *} P<0.001$, compared with control group; $\#$ \# $P$ 0.001, compared with $\mathrm{No} \mathrm{Mg}^{2+}$ group 
occurrence of epilepsy in immature brain is different from that in the adult brain $[17,18]$. As a result of its complex and nonspecific clinical manifestations, TLE is easy to be misdiagnosed or missed $[19,20]$. Recently, EEG and neuroimaging have been widely used in the clinical diagnosis of epilepsy which are important auxiliary ways, but the definite diagnosis is primarily according to a detailed examination of clinical manifestations and a thorough medical history. Therefore, biomarkers may contribute to the accurate clinical diagnosis of epilepsy.

As a result of being stabile in serum, economical, rapid and noninvasive, a number of miRNAs have been identified as potential diagnostic tools for many diseases, including neurodegenerative disorders, such as Parkinson's disease and Alzheimer's disease [5, 6]. The levels of certain miRNAs have been reported to be altered in the blood of epilepsy patients, such as miR-106b-5p, miR130a-3p, and miR-146a-5p, and their diagnostic value for epilepsy has also been confirmed [10]. In the present study, the serum expression level of miR-15a-5p was determined to be decreased in children with TLE. Consistently, the dysregulation of miR-15a-5p has been reported in adult epilepsy patients by Wang et al. [10]. Additionally, miR-15a-5p is also reported to be downregulated in patients with Alzheimer's disease, which shows a remarkable correlation with the occurrence of epilepsy $[11,12]$. These data all suggest the important role of miR-15a-5p in the pathogenesis of neurological disorders, supporting our present results.

Accumulated evidence strongly supports the clinical significance of miR-15a-5p in different types of human diseases. For example, Ramzan et al. have reported that miR-15a-5p is differentially expressed in patients with metabolic syndrome (MetS) compared to healthy individuals, and it is identified as a predictive biomarker for MetS [21]. In acute coronary syndrome (ACS) patients, miR-15a-5p is identified to be at low expression level in patients with ST-segment elevated myocardial infarction (STEMI), and has relatively high discriminatory power for STEMI patients [22]. Another study on colorectal cancer has also suggested that miR-15a-5p is upregulated in tumor compared to normal mucosae, and exhibit dramatic diagnostic value [23]. Clinically, the diagnostic value of miR-15a-5p for TLE was also assessed in TLE children. It was noted that miR-15a-5p was of great value for the diagnosis of TLE in children, with high specificity and sensitivity. We concluded that circulating miR-15a-5p may serve as a promising biomarker for the diagnosis of TLE in children. However, correlation of the expression levels of miR-15a-5p with long-term disease of TLE patients was not detected in the current study. In the future, further researches are needed to explore the clinical value of miR-15a-5p for the evolution of TLE.
The present study further explored the effect of miR$15 a-5 p$ on the biological function of hippocampal neuron. To mimic TLE condition in children, primary hippocampal cells were collected from newborn rats, and cultured in the magnesium-free medium for $3 \mathrm{~h}$. It was noted that the expression level of miR-15a-5p was decreased significantly in hippocampal cells treated in magnesium-free medium, which is consistent with the results obtained in TLE children. The function of miR$15 a-5 p$ in regulating cell behaviors has widely reported. In human hepatocellular carcinoma (HCC), miR-15a-5p is reported to be aberrantly expressed in both HCC cell lines and clinical HCC specimens compared to controls, and overexpression of miR-15a-5p inhibits cell proliferation [24]. Another study by Chen et al. has identified miR-15a-5p to be a new target for osteoarthritis (OA) diagnosis and therapy, and miR-15a-5p may be involved in the pathogenesis of OA through regulating chondrocytes cell progression [25]. It is well established that cell death occurs after epilepsy, but the mechanisms underlying neuronal death remain unclear [26]. In the current study, the effect of miR-15a-5p on the cell viability and apoptosis of hippocampal cells was further investigated, and cell transfection was performed to regulate the expression level of miR-15a-5p in hippocampal cells. The results demonstrated that overexpression of miR-15a-5p significantly enhanced cell viability and inhibited apoptosis, which might be the potential mechanism of the involvement of miR-15a-5p in the progress of children TLE. Although we provided a novel insight on the clinical and functional role of miR-15a-5p in children TLE, further studies are needed to explore the underlying mechanism of the involvement of miR-15a-5p in TLE. Additionally, the current study did not explore the role of miR-15a-5p in different types of TLE, such as neoplastic or non-neoplastic diseases related TLE, which might be interesting and important for future studies.

\section{Conclusion}

Taken together, miR-105a-5p was identified to be downregulated in children with TLE, and overexpression of miR-15a-5p promoted the viability and inhibited the apoptosis of hippocampal cells. The current study provides evidence for miR-15a-5p as a promising biomarker for the diagnosis of children TLE, and the upregulation of miR-15a-5p may have the potential to improve the treatment of TLE.

\section{Abbreviations}

ACS: Acute coronary syndrome; AUC: Area under the curve; DMSO: Dimethyl sulfoxide; HCC: Hepatocellular carcinoma; MetS: Metabolic syndrome; mimic NC: Mimic negative control; miRNAs: microRNAs; MRI: Magnetic resonance imaging; OA: Osteoarthritis; qRT-PCR: Quantitative real-time polymerase chain reaction; ROC: Receiver operating characteristic; SD: Standard deviation; STEMI: ST-segment elevated myocardial infarction; TLE: Temporal lobe epilepsy 


\section{Acknowledgements}

Not applicable.

\section{Authors' contributions}

YF and YL initiated and designed the study, performed the experiment regarding patients, analyzed and interpreted the patient data, and prepared the manuscript. XW performed the experiment regarding animals. FL contributed to design of the study, and the revision of manuscript. All authors read and approved the final manuscript.

\section{Funding}

Not applicable.

\section{Availability of data and materials}

All data generated or analyzed during this study are included in this published article.

\section{Ethics approval and consent to participate}

The protocol of this study was approved by the Ethics Committee of Yidu Central Hospital of Weifang, and written informed consent was collected from each participant.

All animals were treated according to the Guide for the Care and Use of Laboratory Animals of the Institute for Laboratory Animal Research.

\section{Consent for publication}

Written informed consent for publication was obtained from each participant.

\section{Competing interests}

The authors declare that they have no competing interests.

\section{Author details}

'Department of Pediatric Medicine, Yidu Central Hospital of Weifang, Weifang, Shandong 262500, P.R. China. ${ }^{2}$ Department of Medical Image, Yidu Central Hospital of Weifang, Weifang, Shandong 262500, P.R. China. ${ }^{3}$ Department of Emergency Surgery, Weifang People's Hospital, No. 151, Guangwen Street, Weifang, Shandong 261000, P.R. China. ${ }^{4}$ Department of Medical Insurance, Weifang Maternal and Child Health Care Hospital, Weifang, Shandong 261021, P.R. China.

Received: 10 September 2019 Accepted: 26 February 2020

Published online: 08 May 2020

\section{References}

1. Manford M. Recent advances in epilepsy. J Neurol. 2017;264(8):1811-24.

2. Wu X, Wang Y, Sun Z, Ren S, Yang W, Deng Y, et al. Molecular expression and functional analysis of genes in children with temporal lobe epilepsy. J Integr Neurosci. 2019;18(1):71-7.

3. Thom M. Recent advances in the neuropathology of focal lesions in epilepsy. Expert Rev Neurother. 2004;4(6):973-84.

4. Nickels KC, Wong-Kisiel LC, Moseley BD, Wirrell EC. Temporal lobe epilepsy in children. Epilepsy Res Treat. 2012;2012:849540.

5. Cardo LF, Coto E, de Mena L, Ribacoba R, Moris G, Menendez M, et al. Profile of microRNAs in the plasma of Parkinson's disease patients and healthy controls. J Neurol. 2013;260(5):1420-2.

6. Hong H, Li Y, Su B. Identification of circulating miR-125b as a potential biomarker of Alzheimer's disease in APP/PS1 transgenic mouse. J Alzheimers Dis. 2017;59(4):1449-58.

7. Bot AM, Debski KJ, Lukasiuk K. Alterations in miRNA levels in the dentate gyrus in epileptic rats. PLoS One. 2013;8(10):e76051.

8. Li MM, Jiang T, Sun Z, Zhang Q, Tan CC, Yu JT, et al. Genome-wide microRNA expression profiles in hippocampus of rats with chronic temporal lobe epilepsy. Sci Rep. 2014;4:4734.

9. Zhao Q, Yin C, Yuan Y, Zhang H, Teng L. Down-regulation of Mir-145 improves learning and memory abilities in epileptic rats by regulating hippocampal neuron apoptosis. World Neurosurg. 2019;122:e1432-e8.

10. Wang J, Yu JT, Tan L, Tian Y, Ma J, Tan CC, et al. Genome-wide circulating microRNA expression profiling indicates biomarkers for epilepsy. Sci Rep. 2015;5:9522.
11. Satoh J, Kino Y, Niida S. MicroRNA-Seq data analysis pipeline to identify blood biomarkers for Alzheimer's disease from public data. Biomark Insights. 2015;10:21-31

12. Cretin B, Philippi N, Dibitonto L, Blanc F. Epilepsy at the prodromal stages of neurodegenerative diseases. Geriatr Psychol Neuropsychiatr Vieil. 2017;15(1): 75-82.

13. Nunez J. Primary culture of hippocampal neurons from P0 newborn rats. J Vis Exp. 2008;19:e895.

14. Lewis DV, Jones LS, Mott DD. Hippocampal epileptiform activity induced by magnesium-free medium: differences between areas CA1 and CA2-3. Epilepsy Res. 1990;6(2):95-101.

15. Sombati S, Delorenzo RJ. Recurrent spontaneous seizure activity in hippocampal neuronal networks in culture. J Neurophysiol. 1995;73(4):170611

16. Chen Y, Huang X, Chen W, Wang N, Li L. Tenuigenin promotes proliferation and differentiation of hippocampal neural stem cells. Neurochem Res. 2012; 37(4):771-7.

17. Coppola A, Moshe SL. Why is the developing brain more susceptible to status epilepticus? Epilepsia. 2009;50(Suppl 12):25-6.

18. Wahab A, Albus K, Heinemann U. Age- and region-specific effects of anticonvulsants and bumetanide on 4-aminopyridine-induced seizure-like events in immature rat hippocampal-entorhinal cortex slices. Epilepsia. 2011; 52(1):94-103.

19. Sheizaf B, Mazor M, Landau D, Burstein E, Bashiri A, Hershkovitz R. Early sonographic prenatal diagnosis of seizures. Ultrasound Obstet Gynecol. 2007:30(7):1007-9.

20. de Vries JI. Ultrasonographic diagnosis of fetal seizures: a case report and review of the literature. BJOG. 2008;115(1):129-30 author reply 30.

21. Ramzan F, D'Souza RF, Durainayagam BR, Milan AM, Markworth JF, MirandaSoberanis $\mathrm{V}$, et al. Circulatory miRNA biomarkers of metabolic syndrome. Acta Diabetol. 2020;57(2):203-14.

22. Tong KL, Mahmood Zuhdi AS, Wan Ahmad WA, Vanhoutte PM, de Magalhaes JP, Mustafa MR, et al. Circulating MicroRNAs in Young Patients with Acute Coronary Syndrome. Int J Mol Sci. 2018;19(5):1467.

23. Kontos CK, Tsiakanikas P, Avgeris M, Papadopoulos IN, Scorilas A. miR-15a$5 p$, a novel prognostic biomarker, predicting recurrent colorectal adenocarcinoma. Mol Diagn Ther. 2017:21 (4):453-64.

24. Long J, Jiang C, Liu B, Fang S, Kuang M. MicroRNA-15a-5p suppresses cancer proliferation and division in human hepatocellular carcinoma by targeting BDNF. Tumour Biol. 2016;37(5):5821-8.

25. Chen $\mathrm{H}$, Tian Y. MiR-15a-5p regulates viability and matrix degradation of human osteoarthritis chondrocytes via targeting VEGFA. Biosci Trends. 2017; 10(6):482-8.

26. Xie N, Wang C, Lian Y, Wu C, Zhang H, Zhang Q. Puerarin protects hippocampal neurons against cell death in pilocarpine-induced seizures through antioxidant and anti-apoptotic mechanisms. Cell Mol Neurobiol. 2014;34(8):1175-82.

\section{Publisher's Note}

Springer Nature remains neutral with regard to jurisdictional claims in published maps and institutional affiliations.

Ready to submit your research? Choose BMC and benefit from:

- fast, convenient online submission

- thorough peer review by experienced researchers in your field

- rapid publication on acceptance

- support for research data, including large and complex data types

- gold Open Access which fosters wider collaboration and increased citations

- maximum visibility for your research: over $100 \mathrm{M}$ website views per year

At $\mathrm{BMC}$, research is always in progress.

Learn more biomedcentral.com/submission 